\title{
Biomarkers and Depressive Symptoms in Older Women with and without Cognitive Impairment
}

\author{
James R. Hall ${ }^{1,2 *}$, Leigh A. Johnson ${ }^{3}$, Hoa T. Vo ${ }^{1}$, Robert C. Barber ${ }^{2,4}$, A. Scott Winter ${ }^{1}$, \\ Sid E. O'Bryant ${ }^{2,3}$ \\ ${ }^{1}$ Department of Psychiatry, Behavioral Health and Neuroscience, University of North Texas, Health Sciences Center, \\ Fort Worth, USA \\ ${ }^{2}$ Institute of Aging and Alzheimer's Disease Research, University of North Texas, Health Sciences Center, Fort Worth, USA \\ ${ }^{3}$ Department of Internal Medicine, University of North Texas Health Sciences Center, Fort Worth, USA \\ ${ }^{4}$ Department of Pharmacology and Neuroscience, University of North Texas Health Sciences Center, Fort Worth, USA \\ Email: \{ ${ }^{*}$ james.hall, leigh.johnson, hoa.vo, robert.barber, ascott.winter, sid.obryant\}@unthsc.edu
}

Received November 19, 2011; revised January 22, 2012; accepted February 22, 2012

\begin{abstract}
A number of biological markers have been implicated in late life depression with inconsistent results. The present study examined the relationship between several serum based biomarkers and symptoms of depression in a sample of elderly women with $\mathrm{AD}$ or cognitively intact. Methods 171 females (58 with AD and 113 cognitively intact) were recruited from the Longitudinal Research Cohort of the Texas Alzheimer's Research and Consortium (TARC). Stepwise regressions were conducted with GDS total and subscales and a panel of biomarkers (CRP, IL-10, IL-1 $\alpha$, TNF- $\alpha$, ICAM-1, $\mathrm{BDNF}$, and MIF). ApoE4 status was coded (carrier or non-carrier), and the results were analyzed by cognitive status (AD or controls). Results: None of the biomarkers significantly predicted total GDS score for AD cases, controls or sample as a whole. For the Controls, ICAM significantly predicted Dysphoria and level of Apathy. Among AD patients, MIF, ICAM, and CRP, were significantly associated with Apathy. MIF and ICAM were inversely associated with reported Apathy. CRP was positively associated with Apathy. CRP was also positively related to level of perceived Cognitive Impairment. Conclusions: The present study was one of the first to examine biomarkers related to depression symptoms in elderly women with AD and normal controls. For Controls ICAM alone predicted level of apathy. In the AD group, MIF, CRP, and ICAM were significantly associated with apathy. More research examining the relationship between biomarkers and depression is needed in older patients with and without cognitive impairment across genders.
\end{abstract}

Keywords: Biomarkers; Depression; Alzheimer's Disease; Gender

\section{Introduction}

Depression is a debilitating condition and is more prevalent among women than men. Lifetime prevalence rates have been reported as high as $43 \%$ in prospective studies [1]. Late-life depression is responsible for reduced quality of life and increased mortality among the elderly [2]. The exact etiology for depression remains unknown, however, research focusing on women has evaluated fundamental differences in endocrine changes across the menstrual cycle. The cyclic fluctuations across the reproductive cycle have profound influence on a women's mood $[3,4]$. As such, increased levels of progesterone have been associated with increased symptoms of depression. Also in women, reduced levels of serotonin [5] and estrogen [6] have demonstrable affects on symptoms of depression. In older women, other etiologies have been proposed including menopause [7] and cerebrovascular

"Corresponding author. system disturbances [8], particularly for symptoms of apathy.

Biological constituents have a significant role in depression, particularly in women, hence the increased interests in the relationship between late-life depression and biomarkers in older women. It is speculated that a high level of inflammatory cytokines, resulting from systematic, low-grade inflammation, is linked to experiences of depression in elderly women. A number of potential biomarkers have been identified in the literature [9]: IL-6, [10]; IL-10 [11,12]; IL-1 [13]; C-reactive protein (CRP) [14]; tumor necrosis factor (TNF- $\alpha$ ) [15]; and Macrophage migration inhibitory factor (MIF) [16,17]. Additionally, brain-derived neurotropic factor (BDNF) is known to have a role in depression. Although the exact causality remains unclear $[18,19]$, BDNF has been found to be related to the expression of depression in mild $\mathrm{AD}$ patients [20]. Intercellular Adhesion Molecule-1 (ICAM-1) has been more frequently associated with depressive symp- 
toms in vascular models of late-life depression [21-23]. Apolipoprotein E (ApoE $\varepsilon 4$ allele) status has also been associated with level of cognitive impairment in elderly patients [24] with affective states being related to cognition only among non-carriers of the ApoE4 allele [25]. While current research on biomarkers in late-life depression has implicated several potential markers, the relationship between specific markers and specific depressive symptoms has not been investigated.

A number of biomarkers for late-life depression have been implicated in control and patient samples, but results remain inconsistent. Studies have been limited to evaluating depression in either AD or cognitively intact elders; however the examination of markers for late life depression in both normal controls and $\mathrm{AD}$ patients are lacking. Most significantly, prior studies have focused on relationships between cerebrospinal fluid or serum biomarkers and depression in elderly women without attention to subtypes or sub-classification of symptoms. Past research in our laboratory has demonstrated significant value in the use of individual symptom subscales along with global depression scores [26,27] and the importance of gender in understanding the presentation of depressive symptoms and related biological features [28] in the elderly.

The current study addresses these limitations by 1) evaluating both cognitively intact elderly and patients with mild $\mathrm{AD}, 2$ ) assessing both global depression scores and subscale scores in each group, 3) identifying markers that are associated with level of cognitive function and depressive symptoms.

\section{Methods}

\subsection{Sample}

The sample was drawn from the women enrolled in the Longitudinal Research Cohort of the Texas Alzheimer's Research Consortium (TARC) that had complete serum biomarker and depression data. In order to control for disease severity only those women with a Clinical Dementia Rating scale global score of $\leq 1.0$ were included in the sample. The final sample consisted of 171 female, 58 meeting the diagnostic criteria for mild AD and 113 assessed as cognitively intact (normal controls; NC). The methodology of the TARC project has been described in detail elsewhere [29]. Briefly, TARC is a longitudinal multi-site study of a cohort of AD patients and normal controls where each participant undergoes an annual evaluation that includes a medical examination, interview, neuropsychological testing, and blood draw. AD patients met consensus based diagnosis for probable AD based on NINCDS-ADRDA criteria. Normal controls scored within normal limits on neuropsychological testing and had a global CDR of 0 .
Normal controls were on average 67.69 years of age $(\mathrm{SD}=12.07)$ and the AD sample had a mean of 79.25 $(\mathrm{SD}=7.50)$. The control sample had an average of 14.72 (3.348) years of education and the AD sample 13.15 (3.932) years. There was a significant difference on both age $(p=0.001)$ and education $(p=0.001)$ with the AD sample being both older and less educated. The majority of participants were Caucasian (98\%) with black or African American (1.5\%) the next largest group. The TARC project received Institutional Review Board approval and all participants and/or caregivers provided written informed consent.

\subsection{Assessment}

The Geriatric Depression Scale (GDS 30) was administered as part of the TARC neuropsychology core battery. In addition to the GDS, the battery consists of common instruments administered as part of the established Alzheimer's disease clinical/research platforms at each participating institution and includes digit span (WAIS-R, WAIS-III, WMS-R), Trail Making Test, WMS Logical Memory and Visual Reproduction (WMS-R and WMS-III), Boston Naming Test (30- and 60-item versions), verbal fluency (FAS), Clock Drawing Test, the American National Adult Reading Test (AMNART), Mini-Mental State Examination (MMSE), and ratings on the Clinical Dementia Rating scale (CDR).

\subsection{Description of Subscales on the Geriatric Depression Scale}

The Geriatric Depression Scale (GDS-30) [30] was the first depression scale designed specifically for older populations and has been widely used primarily as a screening instrument in clinical and research settings. A recent factor analytic study [31] identified a four-factor solution underlying the scale: dysphoria; meaninglessness; apathy; and cognitive impairment. The dysphoria factor contains 11 items primarily associated with a sad mood. The meaninglessness factor consists of seven items that reflect an appraisal of the meaning (or lack thereof) in one's life. The apathy factor is made up of six items that reflect a lack of motivation or initiative. The cognitive impairment factor consists of six items that reflect difficulty and concern with cognitive processes. The utility of the subscales has been shown in studies relating them to cognitive functioning in $\mathrm{AD}$ and normal elderly [26] and to cognitive functioning in a non-demented rural multi-ethnic sample [32].

\subsection{Assays}

Non-fasting blood samples were collected in serum-separating tubes during clinical evaluations, allowed to clot at room temperature for 30 minutes, centrifuged, aliquoted, 
and stored at $-80^{\circ} \mathrm{C}$ in plastic vials. Batched specimens from either baseline or year-one follow-up exams were sent frozen to Rules Based Medicine (RBM, www.rules basedmedicine.com, Austin, TX) where they were thawed for assay without additional freeze-thaw cycles using the RBM multiplexed immunoassay human Multi-Analyte Profile (humanMAP). Individual proteins were quantified with immunoassays on colored microspheres. Information regarding the least detectable dose (LDD), interrun coefficient of variation, dynamic range, overall spiked standard recovery, and cross-reactivity with other humanMAP analytes can be readily obtained from RBM. IL-6 levels were measured, but were below the detectable range and were not included in the analysis.

\subsection{Data Analysis}

Analyses of the data were performed using SPSS version 17.0. For total GDS score and each of the four GDS subscale scores One-way ANOVA compared cognitively intact and AD females (Table 1). Stepwise regression modeling was used to evaluate the link between total GDS score and each subscale (dysphoria, apathy, meaninglessness, and cognitive impairment) and the biomarker panel by level of cognitive impairment (AD or cognitively intact). Items on the GDS 30 were scored $(0=$ absence and $1=$ presence of symptom) and then added for composite subscale scores. Independent variables for the regression analysis included biomarkers (CRP, IL-10, IL- $1 \alpha$, TNF- $\alpha$, ICAM-1, BDNF, and MIF). Means and standard deviations for the normalized biomarkers for each group are shown in Table 2. ApoE4 status was coded (carrier or non-carrier). Covariates were age and years of formal education. Significance level was set at 0.05 .

\section{Results}

The results of the regression analysis for the entire sample regardless of cognitive status revealed that CRP was associated with level of apathy, $\left(t=-2.973, p<0.003 ; \mathrm{R}^{2}\right.$ $=0.050)$. No other biomarkers were significantly related to the depression variables. Cases and controls were then

Table 1. GDS subscale means and standard deviations.

\begin{tabular}{cccccc}
\hline & GDS 30 & Dys & Mgl & Apathy & CI \\
\hline $\mathrm{NC}$ & 2.81 & 0.69 & 0.27 & 0.92 & 1.03 \\
$(\mathrm{~N}=113)$ & $(3.902)$ & $(1.743)$ & $(0.964)$ & $(1.671)$ & $(1.529)$ \\
& & & & & \\
$\mathrm{AD}$ & 4.82 & 1.34 & 0.66 & 1.36 & 2.07 \\
$\mathrm{~N}=58)$ & $(4.462)$ & $(1.802)$ & $(1.085)$ & $(1.334)$ & $(1.543)$ \\
& & & & & \\
Total & 3.72 & 0.91 & 0.40 & 1.07 & 1.38 \\
$(\mathrm{~N}=171)$ & $(3.759)$ & $(1.479)$ & $(0.857)$ & $(1.2490$ & $(1.347)$ \\
\hline
\end{tabular}

Dys = dysphoria; $\mathrm{Mgl}=$ meaninglessness $; \mathrm{CI}=$ cognitive impairment. analyzed separately. For the NC sample (Table 3), ICAM emerged as a significant predictor of level of Dysphoria $\left(t=2.343, p<0.021 ; \mathrm{R}^{2}=0.047\right)$ and level of Apathy $(t$ $\left.=2.592, p<0.011 ; \mathrm{R}^{2}=0.057\right)$.

Among AD patients, (Table 4) MIF, ICAM, and CRP, were significantly associated with the Apathy. MIF and ICAM were inversely associated with reported Apathy while CRP has a positive relationship with level of Apathy. CRP was also positively related to level of perceived Cognitive Impairment $\left(t=2.383, p=0.021 ; \mathrm{R}^{2}=0.092\right)$. The addition of TNF- $\alpha$ to CRP produced a regression equation that accounted for over $20 \%$ of the variance in the level of perceived Cognitive Impairment.

\section{Discussion}

While the current study is not the first to examine the relationship between serum-based biomarkers and symptoms of depression in cognitively normal and $\mathrm{AD}$ females, it is the first to examine sub-classifications of depressive symptoms. Irrespective of level of cognitive impairment, high levels of CRP predicted apathy in older women. Prior studies have reported similar findings. Bremmer et al. [10] found higher levels of CRP were associated with increased Center for Epidemiological Studies-Depression Scale (CES-D) scores, but that these effects diminished when authors statistical accounted for age and chronic disease status. Results from another mixed gender study, not accounting for level of cognitive impairment, suggested similar results for CRP [33]. Our findings provide similar results, when level of cognitive functioning is not accounted taken into account.

Table 2. Means and standard deviations for normalized values of biomarkers.

\begin{tabular}{ccc}
\hline Biomarker Women & Alzheimer's Women & Cognitively Intact \\
\hline BDNF & $5.0411(0.5971)$ & $4.9013(0.5091)$ \\
CRP & $0.9065(1.7460)$ & $1.3459(1.6340)$ \\
ICAM & $6.9265(1.0075)$ & $7.0845(0.3788)$ \\
IL-1 & $6.4909(1.5878)$ & $6.8711(1.0078)$ \\
IL-10 & $2.7899(1.0075)$ & $3.1809(1.0769)$ \\
MIF & $-3.2492(2.3471)$ & $-3.8881(1.3559)$ \\
TNF $\alpha$ & $1.8713(1.1539)$ & $2.0128(1.2157)$ \\
\hline
\end{tabular}

Table 3. Biomarkers of depressive symptoms for cognitively intact.

\begin{tabular}{|c|c|c|c|c|c|c|}
\hline Scale & Biomarker & $\mathrm{R}^{2}$ & Beta & $p$ & $t$ & 95\% Conf Int \\
\hline GDS 30 & NS & - & - & - & - & - \\
\hline Dys & ICAM & 0.047 & 0.706 & 0.021 & 2.343 & 109 to 1.304 \\
\hline $\mathrm{Mgl}$ & NS & - & - & - & - & - \\
\hline Apathy & ICAM & 0.057 & 0.745 & 0.011 & 2.592 & 0.175 to 1.314 \\
\hline CI & NS & - & - & - & - & - \\
\hline
\end{tabular}

Controls $\mathrm{n}=113$; $\mathrm{NS}=$ no significant biomarkers; Dys $=$ dysphoria; $\mathrm{Mgl}=$ meaninglessness; $\mathrm{CI}$ = cognitive impairment; Conf Int = confidence Interval. 
Table 4. Biomarkers of depressive symptoms for Alzheimer's disease.

\begin{tabular}{|c|c|c|c|c|c|c|}
\hline Scale & Biomarker & $\mathrm{R}^{2}$ & Beta & $p$ & $\mathrm{t}$ & $95 \%$ Conf Int \\
\hline GDS 30 & NS & - & - & - & - & - \\
\hline Dys Mgl & NS & - & - & - & - & - \\
\hline Apathy & NS & - & - & - & - & - \\
\hline Model 1 & MIF & 0.096 & -0.176 & 0.018 & -2.444 & -0.321 to -0.032 \\
\hline \multirow{2}{*}{ Model 2} & MIF & - & -0.356 & 0.001 & -3.637 & -0.552 to -160 \\
\hline & ICAM & 0.194 & -0.588 & 0.013 & -2.576 & -1.046 to -0.131 \\
\hline \multirow{3}{*}{ Model 3} & MIF & - & -0.593 & 0.001 & -3.532 & -0.528 to -0.146 \\
\hline & ICAM & - & -0.567 & 0.013 & -2.562 & -1.011 to -0.123 \\
\hline & CRP & 0.256 & 0.192 & 0.038 & 2.126 & 0.011 to 0.372 \\
\hline \multicolumn{7}{|l|}{$\mathrm{CI}$} \\
\hline Model 1 & CRP & 0.092 & 0.268 & 0.021 & 2.383 & 0.043 to 0.494 \\
\hline \multirow{2}{*}{ Model 2} & CRP & - & 0.369 & 0.002 & 3.282 & 0.144 to 0.595 \\
\hline & TNF- $\alpha$ & 0.203 & -0.471 & 0.008 & -2.765 & -0.812 to -0.129 \\
\hline
\end{tabular}

$\mathrm{AD} \mathrm{n}=58 ; \mathrm{NS}=$ no significant biomarkers; Dys = dysphoria $; \mathrm{Mgl}=$ meaninglessness $; \mathrm{CI}=$ cognitive impairment; Conf Int $=$ confidence Interval

When level of cognitive functioning was considered, ICAM was the only significant predictor of depressive symptoms in cognitively intact females. ICAM has been associated in previous research with apathy, particularly among patients with vascular disturbances [21-23]. The current results suggest that ICAM levels may be useful for predicting depressive symptoms in cognitively intact females. Other research from our group suggests that this association does not hold in a sample of cognitively intact elderly males.

While ICAM alone predicted level of apathy among NC group, MIF and CRP along with ICAM were significantly associated with apathy among mild AD patients. Current finding suggests that the levels of MIF and ICAM are negatively related to level of apathy. While other biomarkers such as ICAM have been linked with symptoms of apathy, MIF has not been useful in predicting apathy in the past. In fact, in a mixed sample report, with a small sample size of ( $\mathrm{n}=18$ patients and 38 normal controls), Lee et al. [16] found that MIF did not contribute any variance in predicting late-life depression. While the role of MIF is unknown in the disease progression of patients with $\mathrm{AD}$ and late-life depression, it has been implicated in depression in a recent animal study [17], suggesting that MIF is inversely related to depression. Our findings suggest that increased MIF levels are negatively associated with depressive symptoms in human females. It is important to note that MIF levels have also been associated with risk for $\mathrm{AD}$ [34] which could account for the finding of a relationship between MIF and depressive symptoms in AD females. However, there was no significant difference between $\mathrm{AD}$ and $\mathrm{NC}$ women on MIF levels $(\mathrm{F}=1.842, p=0.172)$ suggesting that the relationship is not due to disease status alone.

CRP was positively associated with apathy in AD females. The addition of CRP to MIF and ICAM accounts for $25.6 \%$ of the variance in the model. CRP was also significantly associated with perceived levels of cognitive impairment among the AD group. The addition of TNF- $\alpha$ accounted for $20.3 \%$ of the total variance. In a mixed gender sample study, TNF- $\alpha$ was positively associated depressive symptoms in healthy elderly men and women [15]. However, the current finding suggested a negative association in $\mathrm{AD}$ female patients. Current differences may be attributable, at least partly, to effects of mixed gender analysis, which may have diluted significance findings. In addition, methodological differences are also attributable to differences in prior findings.

Limitations to the current study include small and uneven sample sizes. Efforts to replicate the current findings are important for the understanding the role of biomarkers in late-life depression among cognitively impaired and unimpaired elders. Interpretations of current findings should take into consideration its cross-sectional nature and its relatively small sample of individuals with a higher level of depressive symptoms. The overall level of depressive symptoms was low and the small number of patients with at least mild depression reflects a sampling bias in the TARC cohort where individuals with high initial levels of depression were excluded from the study. The current study did not use diagnostic criteria to determine the presence of depressive symptoms but rather relied on scores for a measure of depression. It may well be that individuals with higher levels of symptoms or a 
Major Depressive Disorder may present with a distinct biomarker profile. Another limitation is the absence of IL-6 in the current analysis. While some studies have suggested the significance of IL-6, findings have not been consistent. The application of these scales to a cognitively normal sample may be a limitation although our previous research on cognitively intact elderly using the subscales has shown their utility in describing the relationship of depressive symptom clusters to neuropsychological performance.

The TARC sample is relatively well educated, urban and predominately Caucasian. Among the characteristics of the sample, the AD group was both significantly older and less well educated than the NC. Although both variables were co-varied and did not affect the results, it would be valuable for future research to have a better range of these variables and a better representation of minority populations.

The current study is also unique in that it evaluated across cognitively intact and AD patients. The present research advocates for the importance of continuing to explore inflammatory processes in older patients with and without cognitive impairment across gender. Current data also suggest the value of using subscales rather than global depression scores alone as outcome measures. Despite it limitations, the present study is the first to specifically examine the relationship between females, cognitive status, clusters of depressive symptoms and biomarkers. Additional efforts are warranted in this research area to utilize biomarkers to more accurately predict late-life depression among males and females with varying cognitive capacities and varying levels of depression.

\section{Acknowledgements}

This study was made possible by the Texas Alzheimer's Research Consortium (TARC) funded by the state of Texas through the Texas Council on Alzheimer's Disease and Related Disorders.

\section{REFERENCES}

[1] J. Wiltfang, P. Lewczuk, P. Riederer, E. Grunblatt, C. Hock, P. Scheltens and K. Blennow, "Consensus Paper of the WFSBP Task Force on Biological Markers of Dementia: The Role of CSF and Blood Analysis in the Early and Differential Diagnosis of Dementia," World Journal of Biological Psychiatry, Vol. 6, No. 2, 2006, pp. 69-84. doi:10.1080/15622970510029786

[2] S. E. Starkstein, P. Gustavo, E. Chemerinski and J. Kremer, "Syndromic Validity of Apathy in Alzheimer's Disease," American Journal Psychiatry, Vol. 158, 2001, pp. 872877. doi:10.1176/appi.ajp.158.987.872

[3] H. T. Vo, B. D. Smith and S. Elmi, "Menstrual Endocrinology and Pathology: Premenstrual Syndrome: Caffeine, Physiology, and PMS," In: B. Smith, Ed., Caffeine and
Activation Theory: Effects on Health and Behavior, CRC Press, Boca Raton, 2007, pp. 12-22.

[4] J. Ryan, J. H. Burger, H. C. Szoeke, C. P. Lehert, P. M. L. Ancelin, V. W. Henderson and L. Dennerstein, "A Prospective Study of the Association Between Endogenous Hormones and Depression Symptoms in Postmenopausal Women," Menopause, Vol. 16, No. 3, 2009, pp. 509-517. doi:10.1097/gme.0b013e31818d635f

[5] H. D'haenen, "Imaging the Serotonergic System in Depression," European Achieves of Psychiatry and Clinical Neuroscience, Vol. 251, No. 2, 2001, pp. 76-80. doi:10.1007/BF03035133

[6] J. L. Payne, "The Role of Estrogen in Mood Disorders in Women," International Review of Psychiatry, Vol. 15, 2003, pp. 280-290. doi:10.1080/0954026031000136893

[7] J. T. Bromberger, H. M. Kraitz, Y. F. Chang, J. M. Cyranowski, C. Brown and K. S. Matthews, "Major Depression during and after the Menopausal Transition: Study of Women's Health across the Nation (SWAN)," Psychological Medicine, Vol. 41, No. 9, 2011, pp. 1879-1888. doi:10.1017/S003329171100016X

[8] J. T. Bogousslavsky, "Emotions, Mood, and Behavior after Stroke," Stroke, Vol. 34, No. 4, 2003, pp. 1046-1050. doi:10.1161/01.STR.0000061887.33505.B9

[9] Y. Dowlati, N. Herrmann, W. Swardfager, H. Lui, L. Sham, E. K. Reim and K. L. Lanctot, "A Meta-Analysis of Cytokines in Major Depression," Biological Psychiatry, Vol. 67, No. 5, 2010, pp. 446-457. doi:10.1016/j.biopsych.2009.09.033

[10] M. A. Bremmer, A. T. Beekman, D. J. Deeg, B. W. Penninx, M. G. Dik, C. E. Hack and W. J. Hoogendiijk, "Inflammatory Markers in Late-Life Depression: Results from a Population-Based Study," Journal of Affective Disorders, Vol. 106, No. 3, 2008, pp. 248-255. doi:10.1016/j.jad.2007.07.002

[11] M. Berk, A. A. Wadee, R. H. Kuschke and A. O'NeillKerr, "Acute Phase Proteins in Major Depression," Journal of Psychosomatic Research, Vol. 43, No. 5, 1997, pp. 529-534. doi:10.1016/S0022-3999(97)00139-6

[12] A. Sluzewska, J. Rybakowski, E. Bosmans, M. Sobieska, R. Berghmans, M. Maes and K. Wiktorowicz, "Indicators of Immune Activation in Major Depression," Psychiatry Research, Vol. 64, No. 3, 1996, pp. 161-167. doi:10.1016/S0165-1781(96)02783-7

[13] A. Reichenberg, R. Yirmiya, A. Schuld, T. Kraus, M. Haak, A. Morag and T. Pollmacher, "Cytokine-Associated Emotional and Cognitive Disturbances in Humans," Archives of General Psychiatry, Vol. 58, No. 5, 2001, pp. 445-452. doi:10.1001/archpsyc.58.5.445

[14] H. Moshage, "Cytokines and the Hepatic Acute Phase Response," Journal of Pathology, Vol. 181, No. 3, 1997, pp. 257-266.

doi:10.1002/(SICI)1096-9896(199703)181:3<257::AID-P ATH756>3.0.CO;2-U

[15] E. M. Sternberg, G. P. Chrousos, R. L. Wilder and P. Gold, "The Stress Response and the Regulation of Inflammatory Disease," Annals of Internal Medicine, Vol. 117, No. 10, 1992, pp. 854-866. 
[16] K. S. Lee, J. H. Chung, K. H. Lee, M. J. Shin, B. H. Oh, S. H. Lee and C. H. Hong, "Simultaneous Measurement of 23 Plasma Cytokines in Late-Life Depression," Neurological Science, Vol. 30, No. 5, 2009, pp. 435-438. doi:10.1007/s10072-009-0091-1

[17] C. L. Varea, J. E. Castro, H. Sakouhi-Ouertatani, T. Calandra, H. A. Lashuel and C. Sandi, "Macrophage Migration Inhibitory Factor is Critically Involved in Basal and Flouxetine-Stimulated Adult Hypocampal Cell Proliferation and in Anxiety, Depression, and Memory-Related Behaviors," Molecular Psychiatry, Vol. 16, No. 5, 2010, pp. 543-577.

[18] K. Martinowich, K. H. Manji and B. Lu, "New Insights into BDNF Function in Depression and Anxiety," Nature Neuroscience, Vol. 9, 2007, pp. 1089-1093. doi:10.1038/nn1971

[19] L. Pezawas, A. Meyer-Lindenberg, A. L. Goldman, B. A. Verchinski, G. Chen, B. S. Kolachana and D. R. Weinberger, "Evidence of Biologic Epistasis between BDNF and SLC6A4 and Implications for Depression," Molecular Psychiatry, Vol. 13, 2008, pp. 709-716. doi:10.1038/mp.2008.32

[20] J. R. Hall, S. E. O’Bryant, L. Johnson and R. C. Barber, "Depression and Brain-Derived Neurotrophic Factor Levels in Alzheimer's Disease," Neuroscience and Medicine, Vol. 2, No. 1, 2011, pp. 43-47. doi:10.4236/nm.2011.21006

[21] A. J. Thomas, I. N. Ferrier, R. N. Kalaria, S. A. Woodward, C. Ballard, A. Oakley, R. H. Perry and J. T. O'Brien, "Evaluation in Late-Life Depression of Intracellular Adhesion Molecule-1 Expression in the Dorsolateral Prefrontal Cortex," American Journal of Psychiatry, Vol. 157, No. 10, 2000, pp. 1682-1684. doi:10.1176/appi.ajp.157.10.1682

[22] A. J. Thomas, I. N. Ferrier, R. N. Kalaria, S. Davis and J. T. O'Brien, "Cell Adhesion Molecule Expression in the Dorsolateral Prefrontal Cortex and Anterior Cingulate Cortex in Major Depression in the Elderly," British Journal of Psychiatry, Vol. 181, No. 1, 2002, pp. 129-134.

[23] T. Casoli, G. D. Stefano, M. Balietti, M. Solazzi, B. Giorgetti and P. Fattoreetti, "PeripheralInflammatory Biomarkers of Alzheimer's Disease: The Role of Platelets," Biogerontology, Vol. 11, No. 5, 2010, pp. 627-633. doi:10.1007/s10522-010-9281-8

[24] A. M. Kulminski, S. V. Ukraintseva, I. V. Kulminskaya, K. G. Arbeev, K. C. Land, L. Akushevich and A. L. Yashin, "Cumulative Deficits Better Characterize Susceptibility to Death in Elderly People than Phenotypic Frailty: Lessons from the Cardiovascular Health Study," Journal of American Geriatric Society, Vol. 56, No. 5, 2008, pp. 898-903. doi:10.1111/j.1532-5415.2008.01656.x

[25] M. Edwards, C. Mauer, J. R. Hall, R. C. Barber and S. E.
O'Bryant, "APOE Moderates Link between Affective Status and Cognition: A Project FRONTIER Study," 119th Annual Meeting of the American Psychological Association, Washington DC, August 2011.

[26] J. R. Hall, S. E. O’Bryant, L. A. Johnson and R. Barber, "Depressive-Symptom Clusters and Neuropsychological Performance in Mild Alzheimer's and Cognitively Intact Elderly," Journal of Depression Research and Treatment, 2011, Article ID 396958.

[27] V. Vaccarino, B. D. Johnson, D. S. Sheps, S. E. Ras, S. F. Kelsey, V. Bittner and C. N. Merz, "Depression, Inflammation, and Incident Cardiovascular Disease in Women with Suspected Coronary Ischemia: The National Heart, Lung, and Blood Institute-Sponsored WISE Study," Journal of the American College of Cardiology, Vol. 50, No. 21, 2007, pp. 2044-2050. doi:10.1016/j.jacc.2007.07.069

[28] J. R. Hall, H. Vo, L. Johnson, R. Barber, S. Winter and S. O'Bryant, "Biomarkers and Depressive Symptoms in Cognitively Intact and Alzheimer's Disease Elderly Males," Neuroscience and Medicine, 2011, in press.

[29] S. Waring, S. E. O’Bryant, J. S. Reisch, R. Diaz-Arrastia, J. Knebl and R. Doody, "The Texas Alzheimer's Research Consortium Longitudinal Research Cohort: Study Design and Baseline Characteristics," Texas Public Health Journal, Vol. 63, 2008, pp. 9-13.

[30] J. A. Yesavage, T. L. Brink, T. L. Rose, O. Lum, M. Huang, M. Adey and V. O. Leirer, "Development and Validation of a Geriatric Depression Screening Scale: A Preliminary Report," Journal of Psychiatry Research, Vol. 17, No. 1, 1983, pp. 37-49. doi:10.1016/0022-3956(82)90033-4

[31] J. R. Hall and T. E. Davis, "Factor Structure of the Geriatric Depression Scale in Cognitive Impaired Older Adults," Clinical Gerontology, Vol. 33, No. 1, 2010, pp. 39-48. doi:10.1080/07317110903362127

[32] S. E. O’Bryant, J. R. Hall, K. Cukrowicz, M. Edwards, L. A. Johnson, D. Lefforge and A. Dentino, "The Differential Impact of Depressive Symptom Clusters on Cognition in a Rural Multi-Ethnic Cohort: A Project Frontier Study," International Journal of Geriatric Psychiatry, Vol. 26, No. 2, 2011, pp. 199-205. doi:10.1002/gps.2514

[33] B. W. Penninx, S. B. Kritchevsky, K. Yaffe, A. B. Newman, E. M. Simonsick, S. Rubin and M. Pahor, "Inflamatory Markers and Depressed Mood in Older Persons: Results from the Healthy, Aging and Body Composition Study," Biological Psychiatry, Vol. 54, No. 5, 2003, pp. 566-572. doi:10.1016/S0006-3223(02)01811-5

[34] M. Piccinelli and G. Wilkinson, "Gender Differences in Depression," British Journal of Psychiatry, Vol. 177, 2000, pp. 486-492. doi:10.1192/bjp.177.6.486 